\title{
„Alt werden im Paradies“ - Die ethischen Aspekte der Migration von pflegebedürftigen Menschen
}

\author{
Christine Bally-Zenger $\cdot$ Lisa Eckenwiler $\cdot$ Verina Wild
}

Zusammenfassung Seit einigen Jahren erscheinen in deutschsprachigen Medien Beiträge, die einen neuen Trend in der Versorgung von langzeitpflegebedürftigen Menschen beschreiben: die Migration in ausländische Pflegeheime, insbesondere nach Thailand oder Ost-Europa. Diese Art der Migration wird kontrovers aufgenommen. Einige Medienbeiträge beschreiben diese Praxis u. a. als „Greisen-Export“, „gerontologischen Kolonialismus“ oder ,inhumane Deportation“. Die Begriffe weisen darauf hin, dass diese Migration aus sogenannten High Income Countries in Low and Middle Income Countries aus ethischer Sicht problematisch sein könnte. Allerdings gibt es bislang keine wahrnehmbare wissenschaftliche ethische Auseinandersetzung mit dem Phänomen. In diesem Beitrag diagnostizieren wir, dass es sich bei der Migration Langzeit-Pflegebedürftiger tatsächlich um ein ethisch relevantes Problem handelt, und wir ordnen die von uns identifizierten ethisch relevanten Bereiche unterschiedlichen Ebenen zu: einer individual-, einer gesellschafts-, und einer global-ethischen Ebene. Auf der individualethischen Ebene diskutieren wir Fragen der Autonomie, der Verwandtschaftsbeziehungen, der Rolle von Kultur und Traditionen und der ,guten Pflege“. Auf der gesellschaftsethischen Ebene diskutieren wir strukturelle Herausforderungen der Langzeitpflege und Fragen der sozialen Gerechtigkeit. Auf der globalethischen Ebene verbinden wir unser Thema mit der ethischen Diskussion des Medizintourismus und des Brain Drains und mit Fragen

\footnotetext{
C. Bally-Zenger, M.A. (凸) · Dr. V. Wild

Institut für Biomedizinische Ethik und Medizingeschichte, Universität Zürich,

Winterthurerstrasse 30, 8006 Zürich, Schweiz

E-Mail: christine.bally-zenger@uzh.ch

Dr. V. Wild

Ludwig-Maximilians-Universität München, München, Deutschland

Prof. L. Eckenwiler, PhD

George Mason University, Washington D.C., USA
} 
globaler (Gesundheits-)Gerechtigkeit. Um eine weiterführende normative Analyse vornehmen zu können, sind weitere empirische Daten zu dem Phänomen notwendig.

Schlüsselwörter Migration · Langzeit-Pflege · Angehörige von LangzeitPflegebedürftigen · Gute Pflege · Gerechtigkeit

\title{
"Ageing in paradise" - Ethical aspects of the migration of persons needing long-term care
}

\begin{abstract}
Definition of the problem The German-language media describes a new trend in long-term care: the migration of persons needing such care to nursing homes abroad, especially to Thailand or East Europe. This type of migration is perceived as a controversial practice. Various articles use terms such as "export of the elderly", "gerontological colonialism" or "inhumane deportation". These terms indicate that the migration of persons in need of long-term care from so-called high income countries to low and middle income countries might be problematic from an ethical point of view.

Arguments There has hardly been any academic discussion on this phenomenon. On the basis of the media output and a few scientific articles, we establish that we are dealing with an ethically relevant topic. We then systematically identify relevant ethical aspects. These can be assigned to different levels: an individual-, a societal-, and a global-ethics level. On an individual level we discuss questions of autonomy, relations with relatives, the role of culture and traditions for well-being and best practice in long-term care. On the societal level we discuss structural problems in long-term care, and social justice-related questions. On the global level we connect our topic to the ethics discussion on medical tourism, brain drain and global (health) injustice.

Conclusion In this paper we offer the first, systematic list of relevant ethical issues associated with migration of persons in need of long-term care. In order to proceed with a detailed normative analysis more empirical data on the phenomenon are needed.
\end{abstract}

Keywords Migration - Long-term-care - Relatives of persons in need of longterm-care $\cdot$ Good care $\cdot$ Justice

\section{Einleitung}

Die Weltbevölkerung wird zunehmend älter. 841 Millionen Menschen waren 2013 älter als 60 Jahre, für 2050 prognostiziert die UN über 2 Milliarden (UN 2013). Es ist davon auszugehen, dass die Nachfrage nach Langzeitpflege zunehmen wird, da nicht nur die absolute Zahl der Menschen mit Bedarf ansteigt, sondern mit zunehmendem Alter auch das Risiko für chronische und dementielle Erkrankungen (Prince et al. 2013; WHO 2012; Colombo et al. 2011; Höpflinger et al. 2011; Ferri et al. 2006). 
Langzeitpflege wird meist von weiblichen Angehörigen geleistet (Prince et al. 2013; WHO 2012; Prince und 10/66 Dementia Research Group 2004; Deutscher Ethikrat 2012). Die demografische Entwicklung, aber auch wirtschaftliche und Gerechtigkeitserwägungen führen dazu, dass Frauen vermehrt einer Erwerbsarbeit nachgehen und somit zuhause für die Pflege bedürftiger Angehöriger fehlen (WHO 2002). Hinzu kommt, dass die Angehörigen auf Dauer die Pflege oft nicht alleine gewährleisten können und auf professionelle Pflegedienste angewiesen sind, weil die Betreuung und Pflege umso anspruchsvoller wird, je fortgeschrittener chronische und dementielle Krankheiten sind (Prince et al. 2013; WHO 2012). Die ambulanten oder stationären Pflegedienste gewährleisten also nicht nur adäquate Pflege, sondern ermöglichen es Angehörigen, sich von Belastungen, die durch Pflegetätigkeit entstehen, zu erholen. ${ }^{1}$

In Europa wird einerseits - zum Beispiel durch die Schaffung von Anreizen die Strategie verfolgt, die Pflege durch Angehörige zu fördern (Prince et al. 2013; Colombo et al. 2011). Es ist andererseits jedoch unvermeidlich, professionelle Pflegedienste auszubauen. Dieser Ausbau ist jedoch mit immensen finanziellen Kosten und Personalbedarf verbunden (Prince et al. 2013; UN 2013; WHO 2012; Höpflinger et al. 2011; Ferri et al. 2006).

Die demografischen Entwicklungen, die damit verbundene erhöhte Nachfrage nach qualifiziertem Gesundheitspersonal, die schon heute teilweise nicht mehr ausreichend gedeckt werden kann, und die steigenden Kosten in der Langzeitpflege werden deshalb zunehmend diskutiert (WHO 2002, 2012; Colombo et al. 2011).

Zeitgleich mit den Diskussionen um die Herausforderungen in der Langzeitpflege weisen Medienbeiträge seit einiger Zeit auf einen neuen Trend in der Betreuung und Pflege von betagten und vornehmlich von Demenz betroffenen Personen hin: die Migration dieser Personengruppen in Pflegeinstitutionen nach Ost-Europa und Asien, insbesondere Thailand. Die aufnehmenden Institutionen richten sich werbend an deutschsprachige Langzeitpflegebedürftige.

Die Hauptgründe, die als Vorteil aufgeführt werden, sind die finanzielle Entlastung und die bessere Pflegequalität im Vergleich zum Heimatland. Stormer (2011, S. 43) schreibt: „Für die meisten Betroffenen ist gute Pflege zu teuer und bezahlbare nicht gut genug. Nicht so in Thailand." Connolly (2012a) führt die steigende Anzahl der pflegebedürftigen Migranten ebenfalls auf ,steigende Kosten und abnehmende [Pflege-]Standards“" in den Heimatländern zurück.

Die Berichterstattung bleibt meist nicht neutral und unterstellt dem Trend eine moralische Problematik. So impliziert der Titel „Deutschland, exportiert“ Alte und Kranke in ausländische Pflegeheime“" (Connolly 2012a; Übersetzung durch die Autorinnen) eine Vergegenständlichung der auswandernden Personen. Die Journalistin zitiert eine Wohlfahrtsorganisation, die drastisch von ,inhumaner Deportation“ spricht, und ein Kommentator kritisiert „die Zwangs-Entsorgung der Alten“ als ,gerontologischen Kolonialismus“" (Prantl 2012).

\footnotetext{
${ }^{1}$ Pflegende Angehörige tragen ein erhöhtes Risiko, psychisch zu erkranken. Sie leiden oft an sozialer Isolation und empfinden besonders das Voranschreiten der dementiellen Erkrankung als belastend (Schneider et al. 1999).
} 
Trotz der öffentlich geäußerten moralischen Bedenken wurde der Auswanderungstrend bisher von der wissenschaftlichen ethischen Literatur kaum beachtet. Noch ist unklar, ob diese Art der Migration überhaupt ein ethisches Problem darstellt, welches in einem zweiten Schritt normativ analysiert werden sollte. Hauptziel dieses Artikels ist daher zu diagnostizieren, ob es sich bei der Migration Langzeitpflegebedürftiger tatsächlich um ein ethisch relevantes Problem handelt, und wenn ja, in welcher Hinsicht.

\section{Zahlen und Fakten}

Quellen unserer Analyse sind Zeitungsartikel und audiovisuelle Beiträge auf Deutsch und auf Englisch sowie zwei wissenschaftliche Artikel, die sich jedoch nicht aus ethischer, sondern aus soziologischer Perspektive mit dem Thema befassen (Bender et al. 2014; Horn et al. 2015). ${ }^{2}$

Die Medienbeiträge wurden mit Hilfe einer Internetrecherche gefunden, die im Zeitraum von Anfang 2014 bis Ende 2015 durchgeführt wurde. Für die Suche wurden folgende Stichworte isoliert oder in Kombination, auf Deutsch sowie Englisch verwendet: Migration; Pflege, Langzeitpflege; alt; betagt; Pflegeheim; Demenz; Ausland; Asien; Ost-Europa; Thailand; Ethik; ethisch. In diesem Zeitraum wurden 14 Print- und Online-Artikel in deutscher (Hehli 2015; Vogel 2015; Wuethrich 2015; Gray 2014a, 2014b; Vonlanthen 2013; Schölgens 2013; Winroither und Özkan 2013; Kraushaar 2013; Hanselmann 2013; Siegrist 2013; Dowideit 2012; Prantl 2012; Stormer 2011) und fünf in englischer Sprache (Jorio 2014; Lacey und Foulkes 2014; Kresge 2013; Conolly 2012a, 2012b) gefunden. Zusätzlich fanden wir Dokumentationen (Deutsche Welle 2013; SRF 2011; Müller 2006) und Beiträge in Form einer Nachrichtensendung (SRF 2013a, 2013b), einer Diskussionssendung (ARD 2012), und eines Radio Features (Dorau 2013).

Eine Zusammenstellung aussagekräftiger, quantitativer Daten zu dem Migrationsphänomen ist auf dieser Grundlage nicht möglich. So ist es z. B. schwierig abzuschätzen, wie hoch die Anzahl der Menschen ist, die migrieren, um im Ausland Langzeit-Pflege-Leistungen in Anspruch zu nehmen. ${ }^{3}$ Es ist anzunehmen, dass derzeit eine relativ geringe Anzahl von Menschen - mehrere Tausend - in einem ausländischen Pflegeheim gepflegt wird. Zugleich ist zu vermuten, dass diese Zahl steigen wird. Höpflinger et al. (2011, S. 118) betonen, dass in Zukunft „grenzüberschreitende Angebote (Pflege im Ausland, Pflegende aus dem Ausland) eine verstärkte Bedeutung erhalten." Auch Himmelreicher und Keck (2015) und Horn

\footnotetext{
2 Weitere wissenschaftliche Arbeiten zu der Thematik sind uns nicht bekannt.

${ }^{3}$ Nur Connolly (2012a) nennt Zahlen: "Researchers found an estimated 7,146 German pensioners living in retirement homes in Hungary in 2011. More than 3,000 had been sent to homes in the Czech Republic, and there were more than 600 in Slovakia. There are also unknown numbers in Spain, Greece and Ukraine. Thailand and the Philippines are also attracting increasing numbers." Wir baten die Journalistin mehrfach um Informationen zu ihren Quellen, haben jedoch keine Antwort erhalten.
} 
und Schweppe (2015) und Horn et al. (2015) vertreten die Ansicht, dass in Zukunft betagte Menschen vermehrt migrieren werden. ${ }^{4}$

Bezüglich der Herkunftsländer ist festzustellen, dass in den von uns gefundenen Materialien ausschließlich die Migration von Pflegebedürftigen aus der Schweiz, Deutschland oder Österreich thematisiert wird. Bei den Zielländern beschränken sich manche Beiträge auf die Nennung eines Ziellandes, während andere mehrere erwähnen. Thailand wird am häufigsten (17 von 19) und Osteuropa am zweithäufigsten (7 von 19) genannt. Die erwähnten osteuropäischen Länder sind Ungarn, Tschechien, Slowakei, Polen und die Ukraine. Vereinzelt (3 von 19) werden als Zielländer auch Spanien, Griechenland und die Philippinen genannt. Ob es tatsächlich einen verstärkten Trend der Auswanderung nach Thailand gibt, ist aufgrund der eingeschränkten Datenlage nicht feststellbar. Eine Hypothese ist, dass die Migration nach Thailand aufgrund der Exotik dieser Meldung einen besonderen Reiz ausübt und deshalb überproportional von den Medien aufgegriffen wird.

\section{Darstellung der ethischen Aspekte}

Um die ethisch relevanten Aspekte strukturiert herauszuarbeiten, beginnen wir mit der Darstellung der individualethischen Perspektive. Auf Grundlage des vorhandenen Materials diskutieren wir die Situation urteils- und nicht-urteilsfähiger Personen sowie die Rolle der Angehörigen. Im Anschluss untersuchen wir aus einer gesellschaftsethischen Perspektive die Rolle der Heimatstaaten. Wir fragen, ob eine bestimmte gesellschaftliche Schicht bevorzugt migriert und wenn ja, welche ethische Relevanz dies hätte. Die grenzübergreifende Dimension betrachten wir abschließend aus einer globalethischen Perspektive.

\section{Individualethik I: Die Entscheidungen der urteilsfähigen Pflegebedürftigen}

Es wird mehrfach erwähnt, dass die Entscheidung, in ein ausländisches Pflegeheim auszuwandern, frei und autonom von den Pflegebedürftigen selbst getroffen wird. Sie kann von der Überzeugung getragen sein, dass die Pflege im Ausland bezahlbarer ist und dass Angehörige somit finanziell profitieren. Folgendes Zitat illustriert diesen Sachverhalt: Ein Schweizer ,hat sich für ein Pflegeheim in Ungarn entschieden, weil er nicht sein ganzes Vermögen für die Pflege verschwenden will. ,Ich spare etwa die Hälfte ein'. Seine deutlich jüngere Frau soll doch später noch etwas von seinem Geld haben“" (Hanselmann 2013).

Auch die Qualität der Betreuung spielt eine Rolle. So war es im Falle eines Österreichers ,,seine eigene Entscheidung [in einem ungarischen Pflegeheim zu leben]: Auch wenn es ,ein grosser Schritt` gewesen sei, sei er sehr zufrieden. Die Verpfle-

\footnotetext{
4 Ältere Menschen migrieren meist aus anderen Gründen als Jüngere. Letztere migrieren vor allem, weil sie sich im Zielland bessere Karrierechancen erhoffen (eine Arbeitsstelle oder ein höheres Gehalt). Ältere hingegen migrieren u. a., weil im Zielland die Lebenskosten tiefer, die Infrastruktur besser, das Gesundheitssystem kostengünstiger und besser ist als im Herkunftsland (Zaiceva 2014).
} 
gung sei gut, die Atmosphäre familiär, das Zimmer schön, die Schwestern besonders freundlich" (Winroither und Özkan 2013).

In der westlich geprägten biomedizinischen Ethik - wie auch in der Rechtsprechung - gilt der Grundsatz, dass freie und autonome Entscheide von urteilsfähigen Personen respektiert werden müssen (Beauchamp und Childress 2009; Art. 10 Bundesverfassung der Schweizerischen Eidgenossenschaft; Art. 2 Grundgesetz für die Bundesrepublik Deutschland). So scheint die Migration Langzeitpflegebedürftiger kein ethisch relevantes Problem per se zu sein, wenn sie lediglich aus der individualethischen Perspektive betrachtet wird und wenn ihr eine autonome Entscheidung zugrunde liegt.

Aber wann ist eine solche Entscheidung tatsächlich autonom? In den meisten Medienberichten werden finanzielle Überlegungen als eines der wichtigsten Argumente für die Migration genannt. Zunächst ist eine solche Erwägung als berechtigter Bestandteil einer selbstbestimmen Abwägung anzusehen. Allerdings werden Situationen geschildert, als würden diese Erwägungen den Entschluss zur Auswanderung herbeizwingen. Conolly (2012b) berichtet von I. P., 84, die in einem Pflegeheim in Ungarn lebt und zu ihrem Aufenthalt dort sagt: "'But it is not what I'd have chosen.' Her son [...] said the family was forced to make the decision to take his mother hundreds of miles from her home, saying the state would have demanded his contribution to the costs of her care in Germany, which he says as a small businessman he could have ill afforded."

Es gibt also in den Medienberichten erste Hinweise darauf, dass sich nicht alle frei und selbstbestimmt unter Berücksichtigung aller Vor- und Nachteile für die Pflege im Ausland entscheiden. Spielen tatsächlich zwingende, finanzielle Überlegungen eine zentrale Rolle, dann werden Fragen der sozialen Gerechtigkeit relevant, etwa welche Gesellschaftsgruppen in eine solche Zwangslage kommen. Diese Fragen werden weiter unten ausgeführt. $\mathrm{Zu}$ überlegen ist außerdem, ob sich Personen aus sozialen Gründen gezwungen fühlen könnten auszuwandern, etwa, um ihren Angehörigen nicht zur Last zu fallen. Dass dies eine Rolle spielen könnte, zeigt ein Zitat des oben erwähnten Österreichers: „Meine beiden Söhne sollten ihr eigenes Leben führen“ (Winroither und Özkan 2013).

\section{Individualethik II: Rolle der Angehörigen und Stellvertreterentscheidungen für nicht urteilsfähige Personen}

Die Medien berichten von einer Spezialisierung ausländischer Institutionen auf die Pflege von Personen mit Demenz. Abhängig von der Schwere der Erkrankung kann nicht davon ausgegangen werden, dass sich die betroffene Person selbst zur Migration entscheidet. Ist die Person nicht urteilsfähig und liegt kein vorausverfügter Wille vor, mit der Auswanderung einverstanden zu sein, treffen vermutlich die Angehörigen die Entscheidung. ${ }^{5}$ Diese Tatsache wirft die Frage auf, ob eine solche Entscheidung moralisch angemessen ist.

\footnotetext{
5 Ein Betreiber eines Pflegeheims in Thailand sagte: „Bei den meisten sind es die Angehörigen, die die Entscheidung [im thailändischen Pflegeheim gepflegt zu werden] treffen. Weil sie [die Pflegebedürftigen] nicht mehr entscheiden können, schlichtweg“" (ARD 2012).
} 
Es ist unschwer zu erkennen, dass ein moralisches Problem vorliegt, wenn Angehörige einen nicht urteilsfähigen Menschen in ein ausländisches Pflegeheim verlegen, ohne ihr oder sein bestes Interesse zu verfolgen, sondern stattdessen ein eigennütziges Kosten-Nutzen-Kalkül verfolgen. Der Verdacht liegt nahe, durch solch ein Abschieben entledigten sie sich eines unbequemen Problems. Diese Vorstellung begründet möglicherweise die drastische Begriffswahl wie „Export“ oder „Inhumane Deportation“" (s. oben). Sind solche Fälle empirisch nachweisbar, muss überlegt werden, wie diese Praxis gehandhabt werden soll. Wenn aber solche Fälle empirisch nicht nachweisbar sind, ist es ethisch problematisch, diese Art der Auswanderung als inhumane Deportation zu bezeichnen. Damit könnte eine moralisierende und stigmatisierende Schuldzuweisung an die Angehörigen einhergehen. Vielmehr sollte nach den Gründen gefragt werden, warum jemand sich (aus guten Gründen?) dazu entschließt oder sich (aus strukturellen Gründen?) dazu gezwungen sieht, eine solche Entscheidung zu treffen. Die möglichen guten Gründe werden im Folgenden genauer analysiert, die strukturellen Gründe werden im nachfolgenden Kapitel diskutiert.

Ist eine Person nicht urteilsfähig und liegt kein vorausverfügter Wille vor, so könnten gute Gründe der Angehörigen sein, im besten Interesse der betroffenen Person zu entscheiden. Doch was bedeutet bestes Interesse? Wie und durch wen kann es festgelegt und nachgewiesen werden?

Grundsätzlich ist es im besten Interesse der Betroffenen, keinen Schaden zu erleiden - sondern Nutzen zu erfahren -, der durch das Unterlassen bzw. den Vollzug des Umzugs eintreten würde. Dabei wird in den Medien insbesondere die Qualität der Pflege im Zielland als Argument angeführt. Doch ist diese tatsächlich besser als im Herkunftsland?

Die Aussagen der Angehörigen bezüglich Pflegequalität ähneln sich stets: Der Hauptgrund für die Auswanderung sei die bestmögliche Pflege für ihre Angehörigen. Diese konnten sie im Heimatland entweder nicht finden oder nicht finanzieren. Oft werden die Vorzüge der Pflege in einem ausländischen Pflegeheim den Leistungen einer Institution im Heimatland gegenübergestellt. Während in den Heimatländern das Pflegepersonal ,zu wenig Zeit für zu viele Patienten“ (Stormer 2011, S. 41) hat, ${ }^{6}$ soll die Betreuung im Ausland während $24 \mathrm{~h}$ herzlich, persönlich und individuell auf die Bedürfnisse der Pflegebedürftigen abgestimmt sein. Die Pflege in Thailand wird z. B. so beschrieben: "they [die Pflegebedürftigen] receive constant care on an individual basis. Three caregivers, who take turns accompanying him or her in every activity, look after each patient. At night, there is always someone sleeping in the same room. 'In Switzerland this wouldn't be feasible'" (Jorio 2014).

\footnotetext{
6 Bei einer Befragung des Pflege- und Betreuungspersonals in Pflegeinstitutionen der Schweiz gaben $20 \%$ an: ,,[...] den Bewohner/innen manchmal oder oft keine emotionale Unterstützung bieten zu können. Ein Viertel konnte keine aktivierende Pflege erbringen, [...], dass die Bewohner/innen in der Erhaltung ihrer Selbständigkeit nicht unterstützt wurden. Verwirrte oder kognitiv beeinträchtigte Menschen konnten von einem Fünftel der Befragten nicht genügend beaufsichtigt werden, so dass freiheitseinschränkende Maßnahmen oder beruhigende Medikamente eingesetzt werden mussten“ (Zúñiga et al. 2013, S. 41).
} 
Auch wenn die Anzahl der Pflegenden, die in der Schweiz oder in Deutschland pro Pflegeheimbewohner/-in zur Verfügung steht, kleiner zu sein scheint als in Thailand, ${ }^{7}$ stellt sich die Frage, ob dieses Verhältnis wirklich eines der Hauptindikatoren für gute Pflege ist. Oder grundsätzlich gefragt: Was macht „gute“ oder „,bestmögliche“ Pflege aus? Das Verhältnis zwischen Pflegepersonal pro Anzahl Pflegebedürftiger ist tatsächlich ein Faktor, der die Pflegequalität beeinflusst. Studien zeigen aber, dass die Pflegequalität nicht nur davon abhängt, wie viele Personen eine Pflegefachperson pflegt, sondern auch, wie qualifiziert sie ist (Aiken et al. 2012; Slettebø et al. 2010). In den Medienbeiträgen finden sich keine Informationen, über welche beruflichen Qualifikationen das Pflegepersonal in den ausländischen Pflegeheimen verfügt. Horn et al. (2015, S. 174) halten fest, dass Heime in Thailand wenig reguliert sind und es kaum Vorschriften etwa zur Qualifikation des Personals gibt: "We learned about staffing concepts that do not distinguish between the staff members' duties: they take care of the garden as well of the residents' bodies."

Ein weiteres Thema im Zusammenhang mit bestem Interesse ist die Sprache. In Ungarn, Rumänien, Tschechien oder Polen werden Pflegeheime bevorzugt an Orten gebaut, an denen Teile der Bevölkerung aus historischen Gründen noch deutschsprachig sind (Hanselmann 2013; Kresge 2013; Winroither und Özkan 2013; Dowideit 2012). Es wird betont, dass die Pflegeheime fast durchgehend über deutschsprachiges Personal verfügen. In Thailand werden die fehlenden Deutschkenntnisse des Pflegepersonals - gerade wenn Bewohner an Demenz erkrankt sind - nicht als Nachteil angesehen: „Die sprachliche Distanz wird mit Menschlichkeit überbrückt; Gestik, Mimik, Blickkontakte. Berührungen, kleine Massagen, Händchen halten, Umarmungen, Zärtlichkeiten dienen als Kommunikationsform" (Stormer 2011, S. 43). Es ist unbestritten, dass die beschriebenen Arten der nonverbalen Kommunikation in zwischenmenschlichen Beziehungen wichtig sind - auch in der Pflege. Aber kann diese tatsächlich die verbale Kommunikation ersetzen? Ist der höhere Anteil an nonverbaler Kommunikation im besten Interesse der Pflegebedürftigen? ${ }^{8}$

Einige der Pflegeinstitutionen in Thailand werden von Deutschen oder Schweizern geführt, welche die europäische und thailändische Kultur kennen und beide Sprachen sprechen. Sie nehmen bisweilen die Rolle des Übersetzers zwischen Pflegebedürftigen und Personal wahr. Diese Rolle ist nicht unproblematisch, sind doch der Pflegebedürftige und das Pflegepersonal von einer neutralen und korrekten Übersetzung abhängig. Besonders problematisch erscheint diese Abhängigkeit, wenn wichtige medizinische Entscheidungen z. B. bei lebensverlängernden Maßnahmen getroffen werden müssen. Kann sich der pflegebedürftige Mensch nicht mehr selbst äußern und sind die Angehörigen aufgrund der geografischen Distanz nicht erreichbar, muss der Betreiber unter Umständen ohne Rücksprache entschei-

\footnotetext{
7 Durchschnittlich kommen in einem Schweizer Pflegeheim auf einen Pflegeplatz 0,9 vollzeitäquivalente Arbeitsstellen (BFS 2015).

8 Pflegeheime in den Herkunftsländern rekrutieren zunehmend Personal im Ausland, um den Mangel an inländischem Pflegepersonal zu kompensieren. Diese Entwicklung führt dazu, dass es wegen unzureichender Deutschkenntnisse des ausländischen Personals auch in den Herkunftsländern zu sprachlichen Problemen bei der Pflege kommen kann. Inwiefern diese sprachlichen Schwierigkeiten mit denjenigen in einem ausländischen Pflegeheim vergleichbar sind, und welche Auswirkungen sie auf das beste Interesse der Pflegebedürftigen haben, müsste weiter untersucht werden.
} 
den. Auch Horn et al. (2015, S. 174) halten fest, dass die Betreiber zentrale Akteure mit hoher Entscheidungsgewalt sind, auch bei medizinisch-pflegerischen Entscheidungen: "operators without medical training [...] decide on whether and how much medicine the residents will take - ultimately making decisions on life and death, as they sometimes decide on life prolonging measures." Damit in medizinisch-pflegerischen Belangen dem Wunsch des Patienten entsprechend gehandelt wird, wären Patientenverfügungen ein möglicher Weg. Ob diese verwendet werden, ist uns nicht bekannt.

Auch die Konstanz sozialer Beziehungen und vertrauter kultureller Praktiken ist in eine Nutzen-Schaden-Abwägung einzubeziehen. Es ist nicht eindeutig festzustellen, welchen Stellenwert direkter Kontakt und Gespräche mit vertrauten Personen für Menschen mit Demenz haben. Jedoch lässt der pflegebedürftige Mensch bei einer Auswanderung seine vertrauten Menschen zurück und damit die Möglichkeit, einen Nutzen aus dem direkten Kontakt zu ziehen. Obwohl die Pflegenden im Ausland eventuell mehr Zeit für ihre Patienten haben, können sie die zurückgelassenen Beziehungen wohl nicht ersetzen. Der Ethik-Kodex des International Council of Nurses (ICN 2013, S. 2) fordert kulturelle Sensitivität für eine gelungene Kommunikation, mittels welcher ,die pflegebedürftige Person zeitgerecht die richtige und ausreichende Information auf eine kulturell angemessene Weise erhält, auf die sie ihre Zustimmung zu ihrer pflegerischen Versorgung und Behandlung gründen kann." Wie wichtig kulturelle Verständigung ist, zeigt sich am Beispiel der Musik- und Erinnerungstherapie. Bei dementiell Betroffenen, deren Sprachvermögen eingeschränkt ist, kann vertraute Musik helfen, Erinnerungen und Emotionen wachzurufen und so einen neuen Zugang zu ihrem Selbst zu schaffen. Weil aber biografisch relevante Musik kulturell geprägt ist, sollten Gesundheitsfachpersonen über dieses kulturspezifische Wissen verfügen, damit sie überhaupt in der Lage sind, diesen Zugang herzustellen (Fröhlich-Güzelsoy 2015; Wickel 2004).

Die Anforderung an eine kultursensible „gute Pflege“ in einem thailändischen Pflegeheim zu erfüllen, dürfte mit Schwierigkeiten verbunden sein, denn der Pflegebedürftige und die Pflegefachperson sind in unterschiedlichen kulturellen Kontexten sozialisiert und sprechen unterschiedliche Sprachen. ${ }^{9}$

Auch der Wechsel in einen neuen kulturellen Raum mit anderen Sitten, Riten und Festen ist nicht außer Acht zu lassen. Der Stellenwert der Vertrautheit wird in den Artikeln betont, indem darauf hingewiesen wird, dass ausländische Pflegeheime nur in Betracht gezogen werden sollten, wenn der pflegebedürftige Mensch schon im Laufe seines Lebens eine Beziehung zum Zielland gehabt habe (Jorio 2014; Vonlanthen 2013). Der Deutsche Ethikrat (2012, S. 39) greift das Argument der Vertrautheit ebenfalls auf: „Angesichts der zunehmenden kognitiven Schwächen benötigen Menschen mit Demenz [...] auch eine soziale und emotionale Umgebung, die den Zugang zu den Erkrankten und eine Berücksichtigung ihrer aktuellen Wünsche aufgrund langer Vertrautheit ermöglicht." Es wird zudem betont, dass die Selbstbestimmung der Menschen, die von Demenz betroffen sind, am ehesten gewahrt

\footnotetext{
${ }^{9}$ In Deutschland wird gefordert, kultursensible Pflege von dementiell betroffenen Menschen mit Migrationshintergrund anzubieten. Gesundheitsfachpersonen sollten sowohl über interkulturelle Kompetenz als auch über Sprachkenntnisse ihrer Patient/-innen verfügen (Fröhlich-Güzelsoy 2015; Peters et al. 2014).
} 
werden kann, wenn möglichst wenig äußere Veränderungen stattfinden. Deshalb sei dem Betroffenen ,zu wünschen, dass er so lange wie möglich in seiner gewohnten Umgebung bleiben und weiterhin mit den vertrauten Personen umgehen kann. In der Regel kann alles, was dem Betroffenen erlaubt, in Kontinuität mit seinen Lebensgewohnheiten zu bleiben, Leiden mindern und Pflege erleichtern." (Deutscher Ethikrat 2012, S. 54). Die Schweizerische Alzheimervereinigung (2013) argumentiert ähnlich: „Man kann nicht davon ausgehen, dass es bei Menschen mit fortgeschrittener Demenz keine Rolle spielt, von wem und wo sie gepflegt werden. [...] Erfahrungen zeigen, dass es Demenzkranken besser geht, wenn die Lebensgeschichte in die Pflege einfließt, wenn an frühere Erinnerungen angeknüpft wird und Altes, Bekanntes bestehen bleibt (z. B. mit Möbeln, Musik und typischen Gerichten aus der Zeit, als sie jung waren).“

Selbstverständlich muss möglichen Unzulänglichkeiten der Pflegequalität im Ausland die Qualität im Heimatland gegenüber gestellt werden, weil es an beiden Orten Faktoren gibt, welche die Pflegequalität in positiver oder negativer Weise beeinflussen. Beispielsweise wird erwartet, dass der wachsende Pflegebedarf in Deutschland in Zukunft nicht mehr durch Fachpersonal gedeckt werden kann (s. Deutscher Ethikrat 2012, S. 44 und 45). Um eine Aussage darüber zu treffen, wo und aus welchen Gründen die Pflegequalität tatsächlich den Bedürfnissen der Patienten entspricht und damit auch das ethisch relevante beste Interesse der Pflegebedürftigen besser darstellen zu können - ist mehr empirische Forschung zu Pflegequalität notwendig.

\section{Gesellschaftsethik: Strukturelle Einflussfaktoren in der Langzeitpflege}

Die ökonomischen Rahmenbedingungen haben einen entscheidenden Einfluss auf Art und Weise der Pflege - und zwar auf die stationäre, ambulante und informelle Pflege (Höpflinger et al. 2011, S. 116). In der Schweiz tragen Betroffene einen hohen Anteil der Kosten für die Langzeitpflege selbst. So gehen 63,7\% der gesamten Pflegeheimkosten zu Lasten der pflegebedürftigen Person. Die Krankenkassen übernehmen 20,3\% und die Kantone 15,8\% der Kosten. Im Durchschnitt kostet ein Tag in einem Pflegeheim pro Bewohner/-in 282 CHF (BFS 2015). Auch die Kosten für die Pflegeleistungen der ambulanten Dienste werden zu rund einem Drittel von den Klient/-innen selbst bezahlt. Kosten für hauswirtschaftliche Dienstleistungen wie Mahlzeiten-, Wäsche- oder Putzdienste müssen sie zu $100 \%$ selbst finanzieren (Widmer 2011, S. 104 f.). Ein hoher Anteil der Personen, die ambulante Pflegeleistungen in Anspruch nehmen, bekommt auch Unterstützung von seinem sozialen Umfeld - Angehörige, Nachbarn, Freunde - (Höpflinger et al. 2011, S. 90). Insgesamt wird deutlich, dass die privaten Haushalte im Bereich der Langzeitpflege in finanzieller und personeller Hinsicht stark belastet sind. ${ }^{10}$

In Deutschland stellt sich eine ähnliche Situation dar. 1995 wurde zwar die obligatorische Pflegeversicherung eingeführt, doch ist sie so ausgelegt, dass primär die

\footnotetext{
10 Über $30 \%$ der gesamten Gesundheitsausgaben werden in der Schweiz von den privaten Haushalten getragen. Dabei werden die Haushalte mit niedrigerem Einkommen durch die privaten Gesundheitsausgaben stärker belastet, weil diese Personen im Durchschnitt kränker sind und deshalb höhere Gesundheitsausgaben haben als Personen mit hohem Einkommen (Frey et al. 2011).
} 
Pflegebedürftigen selbst und gegebenenfalls ihre Angehörigen oder die Sozialhilfe die Pflege mitfinanzieren müssen (Da Roit und Le Bihan 2010, S. 291). Das bedeutet, dass u. a. die Kosten für ein Pflegeheim durch die Versicherung nicht vollständig gedeckt sind. Für den Aufenthalt in einem deutschen Pflegeheim, der pro Monat durchschnittlich zwischen 2700 und $3000 €$ kostet, bezahlt die Pflegeversicherung zwischen 1023 bis $1550 €$; in Härtefällen kann dieser Betrag auf bis zu $1918 €$ erhöht werden (Horn et al. 2015, S. 164).

Angesichts der starken Belastung der privaten Haushalte stellt sich die Frage: Inwieweit ist ein Staat rechtlich und ethisch dazu verpflichtet, allen pflegebedürftigen Bürgern einen bezahlbaren Zugang zu qualitativ guten Leistungen des eigenen Gesundheitswesens zu ermöglichen? Die kritischen Stimmen in den Medien, die etwa auf einen inhumanen Greisenexport hinweisen, könnten auch als strukturelle Kritik interpretiert werden. So argumentieren Horn et al. (2015) beispielsweise dafür, dass die strukturellen Probleme der Langzeitpflege in Deutschland - hohe Kosten, Mangel an Pflegepersonal und individueller Pflege - die wesentlichen Gründe dafür sind, dass Pflegeheime im Ausland geschaffen werden. Es könnte also der grundsätzliche Vorwurf an den Staat erhoben werden, es versäumt zu haben, gute und bezahlbare Pflege zu gewährleisten, so dass es nun zu dieser kritischen Problemauslagerung kommt, anstatt vor Ort die Pflegestrukturen zu verbessern.

Aufgrund der starken finanziellen Belastung privater Haushalte und weil finanzielle Überlegungen eine, wenn nicht die zentrale Rolle bei der Migration in ein ausländisches Pflegeheim zu spielen scheinen, werden Fragen der sozialen Gerechtigkeit relevant. Es ist unklar, welchen sozialen Hintergrund die Menschen haben, die im Ausland gepflegt werden. Sind es vor allem Menschen aus ökonomisch schwachen Bevölkerungsschichten, die ausländische Pflegeleistungen nachfragen? Oder migrieren hauptsächlich wohlhabende Personen, weil die Pflege im Heimatland eine geringere Qualität aufzuweisen scheint als im Ausland?

In beiden Fällen stellt sich die gerechtigkeitsrelevante Frage, ob es nicht die Pflicht des Staates ist, allen Bürgern, unabhängig von ihren finanziellen Mitteln, den gleichberechtigten Zugang zu denselben, qualitativ guten Pflegeleistungen zu gewähren? ${ }^{11}$ Diese Frage gewinnt an Brisanz, weil derzeit Gespräche zwischen staatlichen Behörden, Krankenkassen und den Betreibern der ausländischen Pflegeheime stattfinden. Gegenstand dieser Gespräche soll die Übernahme der Kosten für die Leistungen der ausländischen Pflegeheime sein (Hehli 2015; Hanselmann 2013; Winroither und Özkan 2013; Dowideit 2012). Würden die Kosten für die Pflege im Ausland übernommen, müssten die Bedingungen und Kriterien normativ evaluiert werden. Sollten Staaten beispielsweise über den Umfang oder Art von Langzeitleistungen mitbestimmen dürfen, bzw. über Anreizsysteme in die Entscheidungen eingreifen, in welchem Land ein/-e Bürger/-in gepflegt wird? Welche Auswirkungen hätten solche Instrumente auf die soziale Gerechtigkeit? Wichtig ist, dass die Entscheidung nicht nur auf die gepflegte Person und ihr soziales Umfeld einen Einfluss hat, sondern auch auf die Zielländer, in welchen die Pflege stattfindet. Dieser Perspektive wenden wir uns im folgenden Abschnitt zu.

\footnotetext{
${ }^{11}$ Höpflinger et al. (2011) halten fest, dass der Spielraum der wohlhabenden Bevölkerung in der Schweiz
} größer ist, sich diejenigen Pflegeleistungen einzukaufen, die sie sich wünschen, als derjenige der Ärmeren. 


\section{Globale Gerechtigkeit: Welche Auswirkungen hat die Migration auf die Zielländer?}

Aus der Perspektive der globalen Gerechtigkeit ist von Bedeutung, dass Menschen aus sogenannten High Income Countries Gesundheitsleistungen in Low and Middle Income Countries nachfragen. Insbesondere in asiatischen Ländern wie Thailand oder Indien kann diese Art von Migration negative Auswirkungen haben, ähnlich derer, die auch in der Literatur zu Medical Tourism beschrieben werden. Dort wird argumentiert, dass sich zweigeteilte Gesundheitssysteme entwickeln können: So entstehen zwar spezialisierte Privatkliniken für die ausländischen und wohlhabenden einheimischen Patienten, doch verbleibt es zugleich bei einer nur rudimentären medizinischen Grundversorgung für die ländliche und die arme urbane Bevölkerung. Es kommt also zu einer zunehmenden Ungleichheit in der Gesundheitsversorgung. Im wachsenden privaten Sektor wird zudem vermehrt einheimisches Gesundheitspersonal angestellt. Dieser interne Brain Drain ist aus Gerechtigkeitsüberlegungen problematisch, weil dem öffentlichen Gesundheitssektor das Personal für die Versorgung der einheimischen Bevölkerung fehlt (Turner 2013; Connell 2011; Whittaker 2008). Durch den Medizintourismus fließen zwar ausländische Devisen ins Land, die via Steuern der gesamten Bevölkerung zu Gute kommen, doch diese TrickleDown Effekte werden als so gering angesehen, dass sie vernachlässigt werden können (Connell 2011). Vom Medizintourismus profitieren daher derzeit hauptsächlich der private Gesundheitssektor und der Tourismussektor, nicht jedoch die ohnehin weniger privilegierten Bevölkerungsschichten.

Inwieweit diese Argumente auch auf die Migration in ausländische Pflegeheime angewendet werden können, muss untersucht werden. Aufgrund des uns zur Verfügung stehenden Materials können wir festhalten, dass möglicherweise ein ethisch relevantes Problem vorliegt, da das Geschäftsmodell der Langzeitpflegeheime ja gerade darin besteht, in privaten Pflegeheimen einheimische Pflegende anzustellen. Diese Entwicklung scheint besonders problematisch angesichts der auch in Thailand zunehmend alternden Bevölkerung, der Zunahme chronischer Erkrankungen und somit einem auch dort stark steigenden Bedarf an Langzeitpflege (Kespichayawattana und Jitapunkul 2009; Maeseneer et al. 2008). Auch in Thailand werden die Langzeitpflegebedürftigen meist von Familienmitgliedern zuhause gepflegt. Die Pflege innerhalb der Familie wird jedoch zunehmend schwieriger aufgrund der abnehmenden Anzahl an Kindern und deren Erwerbstätigkeit. Thailand steht also ebenfalls vor der großen Herausforderung, in den nächsten Jahren Langzeitpflege-Infrastrukturen für die eigene Bevölkerung aufzubauen (Kespichayawattana und Jitapunkul 2009; Maeseneer et al. 2008).

\section{Zusammenfassung und Schlussfolgerung}

Ziel dieses Artikels war zu diagnostizieren, ob es sich bei der Migration von Langzeitpflegebedürftigen um ein ethisch relevantes Problem handelt und wenn ja, die damit zusammenhängenden ethischen Aspekte zu identifizieren. 
Die Untersuchung der Medienbeiträge und der wissenschaftlichen Artikel zeigt, dass es ethische Aspekte gibt, die bei einer noch zu leistenden normativen Analyse berücksichtigt werden sollten.

Die individuelle Entscheidung zur Auswanderung wird bei urteilsfähigen Personen oft als autonom dargestellt. Weil aber finanzielle Zwangslagen die Entscheidung mitbeeinflussen können, ist unklar, wie autonom sie tatsächlich ist. Zudem ist zu untersuchen, aus welchen Gründen Angehörige entscheiden, eine pflegebedürftige urteilsunfähige Person in einem ausländischen Pflegeheim pflegen zu lassen. Wir haben ein Abschiebeverhalten der Angehörigen als ethisch problematisch identifiziert, welches nicht im besten Interesse der Pflegebedürftigen ist. Allerdings fehlen Daten darüber, ob dieses tatsächlich existiert. Bei der Frage nach dem besten Interesse und der bestmöglichen Pflege haben wir den Verlust der sozialen Beziehungen und der vertrauten Riten, Sprache und Traditionen thematisiert.

Auf der gesellschaftlichen Ebene haben wir herausgearbeitet, dass womöglich ein strukturelles Problem vorliegt und zwar das staatliche Versäumnis, gute und bezahlbare Pflege für alle bereitzustellen. Die Migration von Langzeitpflegebedürftigen ins Ausland weist womöglich auf gerechtigkeitsrelevante Bedingungen im Heimatland hin, die gewisse Bevölkerungsgruppen benachteiligen oder bevorzugen.

Aus globalethischer Perspektive haben wir herausgearbeitet, dass Argumente aus der Debatte des Medizintourismus auch auf die Pflege der Langzeitpflegebedürftigen im Ausland zutreffen könnten. In den Zielländern kann es zu dem folgenden negativen Effekt kommen: zunehmende Gesundheitsungleichheit der einheimischen Bevölkerung durch einen wachsenden privaten Sektor im Gesundheitswesen, der zu einem internen Brain Drain und einer nur rudimentären Versorgung der ärmeren Bevölkerungsschichten führen kann.

Weitere ethische Aspekte können aus dem von uns untersuchten Material nicht abgeleitet werden, es stellen sich aber zusätzliche Fragen: Manifestiert eine Auslagerung der Pflege in Low and Middle Income Countries (LMICs) problematische Strukturen der Geschlechtergerechtigkeit, weil Frauen in LMICs in weniger gut bezahlten Pflegeberufen bleiben, während die Angehörigen in High Income Countries (HICs), die von der Pflege entlastet sind, sich höher bezahlten Berufen zuwenden können? Oder bietet die Pflege von gut zahlenden Klienten in LMICs gerade eine Möglichkeit - auch für Frauen - wirtschaftlich lukrative Geschäfte aufzubauen? Außerdem stellt sich die Frage nach den Konsequenzen, sollte sich die Migration von Langzeitpflegebedürftigen in empirischen Studien als ethisch problematisch erweisen. In welcher Weise dürfen Staaten darauf Einfluss nehmen, unter welchen Umständen und wohin Langzeitpflegebedürftige migrieren?

Wir legen mit unserer Analyse keine vollständige Liste ethisch relevanter Aspekte vor, sondern beginnen damit, strukturiert herauszuarbeiten, welche Aspekte eine genauere Untersuchung verdienen. Damit diese einer normativen Analyse unterzogen werden können, besteht zunächst das Desiderat, das Phänomen der Migration von Langzeitpflegebedürftigen empirisch zu erforschen. Gerade in Anbetracht der demografischen Entwicklung und der steigenden Nachfrage nach Langzeitpflege erscheint es dringend notwendig, Daten zu erheben, die es erlauben, dieses neue Phänomen empirisch und normativ zu verstehen. 


\section{Einhaltung ethischer Richtlinien}

Interessenkonflikt C. Bally-Zenger, L. Eckenwiler und V. Wild geben an, dass kein Interessenkonflikt besteht.

Ethische Standards Dieser Beitrag beinhaltet keine von den Autoren durchgeführten Studien an Menschen oder Tieren.

\section{Literatur}

Aiken LH, Sermeus W, Van den Heede K (2012) Patient safety, satisfaction, and quality of hospital care: cross sectional surveys of nurses and patients in 12 countries in Europe and the United States. BMJ 344:e1717

ARD (2012) Kostenfaktor Oma - wird Pflege unbezahlbar? Günther Jauch. http://www.alzheimerthailand. com/AlzheimerThailand/fernseh-dateien/videofenster66.htm. Zugegriffen: 11. Apr. 2016

Beauchamp TL, Childress JF (2009) Principles of biomedical ethics, 6. Aufl. Oxford University Press, Oxford

Bender D, Hollstein T, Horn V et al (2014) Old age care facilities and care-seeking elderly on the move. Transnatl Soc Rev 4:290-293

Bundesamt für Statistik (2015) BFS Aktuell: Indikatoren der Pflegeheime 2013. http://www.bfs.admin.ch/ bfs/portal/de/index/news/publikationen.Document.197245.pdf. Zugegriffen: 11. April 2016

Colombo F, Llena-Nozal A, Mercier J, Tjadens F (2011) Help wanted? Providing and paying for longterm care. OECD Health Policy Studies. https://www.oecd.org/els/health-systems/47836116.pdf. Zugegriffen: 2. Sep. 2016

Connell J (2011) A new inequality? Privatisation, urban bias, migration and medical tourism. Asia Pac Viewp 52:260-271

Connolly K (2012a) Germany "exporting" old and sick to foreign care homes. http://www.theguardian. com/world/2012/dec/26/german-elderly-foreign-care-homes. Zugegriffen: 11. April 2016. (The Guardian)

Conolly K (2012b) Germany's far-flung pensioners living in care around the world. http://www. theguardian.com/world/2012/dec/28/germany-pensioners-living-care-world. Zugegriffen: 11. April 2016. (The Guardian)

Deutsche Welle (2013) Deutsche Rentner in Osteuropa. https://www.youtube.com/watch?v=uI96d01zT9g. Zugegriffen: 11. April 2016

Deutscher Ethikrat (2012) Demenz und Selbstbestimmung. Stellungnahme. http://www.ethikrat.org/ dateien/pdf/stellungnahme-demenz-und-selbstbestimmung.pdf. Zugegriffen: 2. Sep. 2016

Dorau F (2013) Über die Pflege europäischer Demenzkranker in Thailand. Passage, Schweizer Radio und Fernsehen SRF. http://www.srf.ch/sendungen/passage/ueber-die-pflege-europaeischerdemenzkranker-in-thailand. Zugegriffen: 11. April 2016

Dowideit A (2012) Pflegenotstand: Wenn deutsche Omas in die Slowakei fliehen müssen. http://www. welt.de/wirtschaft/article110328510/Wenn-deutsche-Omas-in-die-Slowakei-fliehen-muessen.html. Zugegriffen: 11. April 2016. (Welt)

Ferri CP, Prince M, Brayne C et al (2006) Global prevalence of dementia: a Delphi consensus study. Lancet 366:2112-2117

Frey M, Liechti D, Feer M (2011) Direkte Gesundheitsausgaben der privaten Haushalte: Out-Of-PocketZahlungen und Kostenbeteiligungen in der Schweiz und im internationalen Vergleich. Schweizerisches Gesundheitsobservatorium. http://www.obsan.admin.ch/sites/default/files/publications/2015/ obsan_dossier_17.pdf. Zugegriffen: 2. Sep. 2016

Fröhlich-Güzelsoy L (2015) Musik- und Erinnerungstherapie bei demenziell erkrankten Patient*innen mit Migrationsgeschichte. Ethische Desiderate der Demenzbehandlung. In: Frewer A, Bergemann L, Schmidhuber M (Hrsg) Jahrbuch Ethik in der Klinik, Bd. 8. Königshausen \& Neumann, Würzburg, S 73-95

Gray DD (2014a) Wenn Alzheimer-Kranke in Thailand Pflege finden. http://www.welt.de/gesundheit/ article123512788/Wenn-Alzheimer-Kranke-in-Thailand-Pflege-finden.html. Zugegriffen: 11. April 2016. (Welt) 
Gray DD (2014b) Alzheimer-Kranke im sonnigen Thailand pflegen. http://www.20min.ch/wissen/news/ story/Alzheimer-Kranke-im-sonnigen-Thailand-pflegen-24040593. Zugegriffen: 11. April 2016. (20minuten)

Hanselmann L (2013) Schweizer sollen in Ungarn ins Billig-Altersheim. http://www.20min.ch/schweiz/ news/story/Schweizer-sollen-in-Ungarn-ins-Billig-Altersheim-13655050. Zugegriffen: 11. April 2016. (20minuten)

Hehli S (2015) Schweizer Demenzresort im tropischen Gegenwind. http://www.nzz.ch/schweiz/schweizerdemenzresort-im-tropischen-gegenwind-1.18569648. Zugegriffen: 11. April 2016. (Neue Zürcher Zeitung)

Himmelreicher R, Keck W (2015) Transnational aging as reflected in Germany's pension insurance. In: Horn V, Schweppe C (Hrsg) Transnational aging: current insights and future challenges. Routledge, New York London, S 107-125

Höpflinger F, Oglesby-Bayer L, Zumbrunn A (2011) Pflegebedürftigkeit und Langzeitpflege im Alter. Aktualisierte Szenarien für die Schweiz. Hans Huber, Bern

Horn V, Schweppe C (2015) Transnational aging: current insights and future challenges. In: Horn V, Schweppe C (Hrsg) Transnational aging: current insights and future challenges. Routledge, New York London, S 1-15

Horn V, Schweppe C, Bender D, Hollstein T (2015) Moving (for) elder care abroad. In: Horn V, Schweppe C (Hrsg) Transnational aging: current insights and future challenges. Routledge, New York London, S $163-177$

International Council of Nurses (ICN), Deutscher Berufsverband für Pflegeberufe (2012) ICN-Ethikkodex für Pflegende. https://www.dbfk.de/media/docs/download/Allgemein/ICN-Ethikkodex-2012deutsch.pdf. Zugegriffen: 13. Jan. 2017

Jorio L (2014) Swiss Alzheimer's patients find home in Thailand, Swissinfo: http://www.swissinfo.ch/ eng/offshoring-care_swiss-alzheimer-s-patients-find-home-in-thailand/38281642. Zugegriffen: 11. April 2016

Kespichayawattana J, Jitapunkul S (2009) Health and health care system for older persons. Ageing Int $33: 28-49$

Kraushaar B (2013) Demenz und Burnout: Kranke sollen zur Pflege nach Thailand. http://www. schweizamsonntag.ch/ressort/aktuell/2966/?fb_comment_id=504678536247817_5145515\# f1d229a4c. Zugegriffen: 11. April 2016. (Schweiz am Sonntag)

Kresge N (2013) Germany is exporting its grandmas, Bloomberg L.P.: http://www.bloomberg.com/news/ articles/2013-09-26/germany-exports-its-seniors-to-nursing-homes-abroad. Zugegriffen: 11. April 2016

Lacey A, Foulkes I (2014) Exporting Grandma to care homes abroad. http://www.bbc.com/news/health25438325. Zugegriffen: 11. April 2016. (BBC News)

Maeseneer JD, Moosa S, Pongsupap Y, Kaufman A (2008) Primary health care in a changing world. Br J Gen Pract 58:806-809

Müller C (2006) Der Lauf des Lebens. Margrit Woodtli: geboren in Münsingen, gestorben in Thailand. Dok Schweizer Radio und Fernsehen SRF

Peters T, Grützmann T, Bruchhausen W et al (2014) Empfehlungen zum Umgang mit Interkulturalität in Einrichtungen des Gesundheitswesens. Ethik Med 26:65-75

Prantl H (2012) Die verrückte Idee vom Greisen-Export. http://www.sueddeutsche.de/politik/pflegeheimeim-ausland-die-verrueckte-idee-vom-greisen-export-1.1512615. Zugegriffen: 11. April 2016. (Süddeutsche Zeitung)

Prince M, 10/66 Dementia Research Group (2004) Care arrangements for people with dementia in developing countries. Int J Geriatr Psychiatry 19:170-177

Prince M, Prina M, Guerchet M (2013) World Alzheimer report 2013. Journey of caring. An analysis of long-term care for dementia. Alzheimers's Disease International, London

Da Roit B, Le Bihan B (2010) Similar and yet so different: cash for care in six European countries' long term care policies. Milbank Q 88:286-309

Schneider J, Murray J, Banerjee S, Mann A (1999) EUROCARE: a cross national study of co resident spouse carers for people with Alzheimer's disease: I - factors associated with carer burden. Int J Geriatr Psychiatry 14:651-661

Schölgens G (2013) Pflege in Thailand - was muss ich beachten. http://www.ksta.de/wirtschaft/-auslandpflege-thailand-demenzkranke-alzheimer-auswandern-rente-1705434. Zugegriffen: 11. April 2016. (Kölner Stadtanzeiger) 
Schweizer Radio und Fernsehen SRF (2011) Fortsetzung folgt - Der Lauf des Lebens. http://www.srf. $\mathrm{ch} /$ play/tv/dok-fortsetzung-folgt/video/dok-fortsetzung-folgt-vom-15-04-2011?id=de5daed7-ecfd4b5d-a907-5bd8337b4387. Zugegriffen: 11. April 2016

Schweizer Radio und Fernsehen SRF (2013a) Pflegeheim für Demenzkranke im Urlaubsparadies. http://www.srf.ch/play/tv/10vor10/video/pflegeheim-fuer-demenzkranke-im-urlaubsparadies? id=26a6d48a-da5d-495f-b7a4-7417e7047682. Zugegriffen: 11. April 2016. (10vor10)

Schweizer Radio und Fernsehen SRF (2013b) Demenzheime in Thailand: Paradies wider Willen. http:// www.srf.ch/play/tv/10vor10/video/demenzheime-in-thailand- paradies- wider- willen?id=468fcaf16cca-41a0-9260-648beb85cc7d. Zugegriffen: 11. April 2016. (10vor10)

Schweizerische Alzheimervereinigung (2013) Heim für Demenzkranke in Thailand - Kommentar der Schweizerischen Alzheimervereinigung. http://www.alz.ch/index.php/medienmitteilungen. html?file=tl_files/PDFs/PDF-D\%20Medienmitteilungen/Heim\%20fuer\%20Demenzkranke\%20in $\% 20$ Thailand $\% 20-\% 20 \mathrm{Kommentar} \% 20 \mathrm{der} \% 20$ Schweizerischen\%20Alzheimervereinigung.pdf. Zugegriffen: 2. Sep. 2016

Siegrist N (2013) Vergessen im Paradies. Schweiz Illus 12:52-57

Sletteb $\varnothing$ A, Kirkevold M, Andersen B et al (2010) Clinical prioritizations and contextual constraints in nursing homes - a qualitative study. Scand J Caring Sci 24:533-540

Stormer C (2011) Dement unter Palmen. Enorm 02:38-45

Turner L (2013) Transnational medical travel. Ethical dimensions of global healthcare. Camb Q Healthc Ethics 22:170-180

United Nations (2013) World population ageing. http://www.un.org/en/development/desa/population/ publications/pdf/ageing/WorldPopulationAgeing2013.pdf. Zugegriffen: 2. Sept. 2016

Vogel S (2015) Warum immer mehr Deutsche in Thailand alt werden wollen. http://www.berliner-zeitung. de/panorama/pflege-fuer-alte-und-demenzkranke-warum-immer-mehr-deutsche-in-thailand-altwerden-wollen-1212848. Zugegriffen: 11. April 2016. (Berliner Zeitung)

Vonlanthen D (2013) Ausgelagert ins Land des Lächelns. Sind Demenz-Resorts in Thailand eine Alternative zu Heimen in der Schweiz? Curaviva 6:27-30

Whittaker A (2008) Pleasure and pain: medical travel in Asia. Glob Public Health 3:271-290

Wickel HH (2004) Musik als Emotionsauslösendes Medium in der Biographiearbeit mit älteren Menschen. http://www.st-marienwoerth.de/fileadmin/user_upload/PDF_Ordner/Biografiearbeit-VortragProf.Dr. Wickel.pdf. Zugegriffen: 2.Sep. 2016

Widmer W (2011) Das Gesundheitswesen der Schweiz. Ein Überblick aus individueller, betrieblicher und gesellschaftlicher Sicht. Careum Verlag, Zürich

Winroither E, Özkan D (2013) Altenpflege: Opa wohnt jetzt in Ungarn. http://diepresse.com/home/ panorama/oesterreich/1382748/Altenpflege_Opa-wohnt-jetzt-in-Ungarn?from=suche.intern.portal. Zugegriffen: 11. April 2016. (Die Presse)

World Health Organization (2002) Ethical choices in long-term care: what does justice require. http://www. who.int/mediacentre/news/notes/ethical_choices.pdf. Zugegriffen: 2. Sep. 2016

World Health Organization (2012) Dementia: a public health priority. http://apps.who.int/iris/bitstream/ 10665/75263/1/9789241564458_eng.pdf. Zugegriffen: 2. Sep. 2016

Wuethrich C (2015) „Das Resort müsste in der Schweiz stehen“. http://www.tagblatt.ch/ostschweiz-amsonntag/hintergrund/Das-Resort-muesste-in-der-Schweiz-stehen;art304162,4121987. Zugegriffen: 11. April 2016. (Tagblatt)

Zaiceva A (2014) The impact of aging on the scale of migration. IZA World of Labor. http://wol.iza.org/ articles/impact-of-aging-on-scale-of-migration-1.pdf. Zugegriffen: 2. Sep. 2016

Zúñiga F, Ausserhofer D, Serdaly C et al (2013) Schlussbericht zur Befragung des Pflege- und Betreuungspersonals in Alters- und Pflegeinstitutionen der Schweiz. Universität Basel. https://nursing.unibas. ch/fileadmin/pflege/redaktion/Forschung/SHURP_Schlussbericht_rev_geschuetzt.pdf. Zugegriffen: 11. Jan. 2017 